\title{
Battis/Krautzberger/Löhr, Baugesetzbuch
}

\author{
Inhaltsübersicht
}

\author{
A. Worüber berichtet wird \\ B. Drei Autoren finden sich \\ I. Der Anlaß \\ II. Erst vier, dann drei \\ C. Das Konzept für die gemeinsame Kommentierung \\ I. Das „Leitbild“ \\ II. Die Aufteilung \\ III. Die Abstimmung \\ IV. Der Name \\ D. Der Verlag \\ E. Das Lektorat \\ F. Die Umwelt \\ I. Zehn Auflagen \\ II. Das BauGB 1987 \\ III. Der Einigungsvertrag 1990 \\ IV. Neue städtebaurechtliche Strukturfragen - die Novellen 1983 und \\ 1998 \\ V. Umwelt- und Europarecht - die Novellen 2001, 2004 und 2007 \\ G. Die Entwicklung \\ H. Einige Erfahrungen \\ I. Wem macht Kommentarschreiben eigentlich Freude? \\ II. Kommentarschreiber im Bundesministerium
}

\section{A. Worüber berichtet wird}

Am besten beginne ich mit den ersten Zeilen der Erstauflage von „Battis/Krautzberger/Löhr“", in dem das Konzept der Neuerscheinung knapp umrissen wurde:

„Der hiermit vorgelegte Kommentar zum Bundesbaugesetz ist vornehmlich für die Praxis und für die Ausbildung geschrieben. Er will in verläßlicher, gestraffter und verständlicher Weise denjenigen die notwendigen Informationen zum geltenden Recht vermitteln, die sich ,täglich“ oder nur gelegentlich mit dem Bundesbaugesetz befassen, den Praktikern in Städten, Gemeinden und Kreisen sowie in den staatlichen Behörden, den Richtern und den Rechtsanwälten, den Bauherren und den Architekten, besonders den Studierenden an Universitäten und Fachhochschulen."

Soweit aus dem Vorwort der 1. Auflage von „Battis/Krautzberger/Löhr“ vom Mai 1984. Ein neuer Kommentar in der „Gelben Reihe“ des Verlagshauses C. H. Beck. Warum damals ein neues Buch? Warum gerade diese drei Autoren damals mit den Ortsangaben „Hagen, Bonn und Meckenheim“ - und mit wel- 
chem Konzept? Und wie wurde das über die Jahre bis zur jetzt - mehr als 20 Jahre nach der Erstauflage erscheinenden - 10. Auflage gestaltet, koordiniert und fortentwickelt? Darüber soll im folgenden berichtet werden - mit keinen anderen Quellen als dem Gedächtnis, der Rück-Vergewisserung bei Ulrich Battis und RolfPeter Löhr und den Vorworten der zehn Auflagen.

\section{B. Drei Autoren finden sich}

\section{Der Anlaß}

Von wem ging die Initiative aus? Es waren die beiden im damaligen Bundesbauministerium tätigen Autoren Löhr und Krautzberger, die sich Anfang der achtziger Jahre in einem neu eingerichteten „Grundsatzreferat“ für das Städtebaurecht zusammenfanden, in der Gesetzgebung - im Vergleich zu manchen Kollegen mit großen Namen, allen voran das Urgestein Werner Bielenberg, aber auch Walter Dyong und der etwa gleichaltrige, aber schon seit mehreren Jahren in der Städtebaugesetzgebung tätige Wilhelm Söfker - eher unbeschriebene Blätter, um nicht zu sagen: in der Städtebaugesetzgebung unerfahren. Was tut man da, ist man in einem solchen Umfeld von Wissensträgern, ist jung und nicht ohne Ehrgeiz? Man schreibt über das, was man nun gestalten muß, ein Buch. Unser Motiv war klar: In diesem Arbeitsbereich des Ministeriums, geprägt auch noch von Werner Ernst und Ernst Zinkahn, hatte - so unser Eindruck - eigentlich jeder unserer Kollegen etwas veröffentlicht, eine Schreibkultur, in der wir uns als Neulinge wiederfanden. Und da wir nun beide das Grundsatzreferat waren - von der damaligen politischen Leitung des Ministeriums der Städtebauabteilung eher etwas von oben implantiert - mußten wir etwas tun: Uns rasch einarbeiten, Kompetenz und Profil gewinnen. Also: ein Buch schreiben, einen Kommentar.

Rolf-Peter Löhr erinnert sich: „Wie genau es zu dem Kommentar gekommen ist, weiß ich nicht. Ich bin nur ziemlich sicher, daß wir darüber gesprochen haben, wie wir uns als Neulinge im Baurecht in der Fachwelt bekannt machen können und gleichzeitig Kompetenz demonstrieren. Dazu ist mir sicher, wie immer bei solchen Gelegenheiten, die Aussage von Schmidt-Aßmann ${ }^{1}$ eingefallen, daß, wenn man auf einem Gebiet keine Ahnung hat, man darüber einen wissenschaftlichen Aufsatz publizieren müsse. Nur so lerne man intensiv und ernsthaft genug."

Das sah ich genauso, wobei Rolf-Peter Löhr tiefstapelte, hatte er sich doch immerhin mit einer übrigens wegweisenden Arbeit über die Flächennutzungsplanung promoviert, während ich zum Baurecht nur einige Aufsätze vorweisen konnte. Aber allein die Qualen des Schreibens eines Buchs decken alles auf, das was man bei Vorträgen und selbst bei Aufsätzen leicht überspielen kann. So sind wir irgendwie gemeinsam auf den Kommentar gekommen.

${ }^{1}$ Löhr kam als Wiss. Assistent von Prof. Dr. Eberhard Schmidt-Aßmann von der Ruhr-Universität Bochum ins Ministerium. 


\section{Erst vier, dann drei}

Da das Städtebaurecht, damals noch hauptsächlich das BBauG, ein weites Feld war, haben wir nach Mitstreitern gesucht. Dazu ist Rolf-Peter Löhr Prof. Dr. Ernst Pappermann eingefallen, den er gut kannte und der einschlägige Publikationserfahrungen mit dem Verlag C.H. Beck hatte. ${ }^{2}$ Er war bereit mitzumachen. Und er war es auch, der die Kontakte zum Verlag hergestellt hat. Er hatte auch die Idee, daß neben der Ministerialinstanz und ihm als kommunalem Vertreter, ein profilierter, jüngerer Wissenschaftler gewonnen werden sollte. Da er damals als Hauptgeschäftsführer des Deutschen Städtetags ausgewählt war, hielt er die Erweiterung des Teams auch aus arbeitsökonomischen Gründen für notwendig. So hat er Ulrich Battis vorgeschlagen, der seinerzeit an der Fernuniversität Hagen wirkte. Ich hatte es übernommen, Ulrich Battis, den ich persönlich so wenig wie Ernst Pappermann kannte, zu einem gemeinsamen Gespräch einzuladen. Auch Ulrich Battis kannte Rolf-Peter Löhr und mich nur aus der Literatur und aus unserer Aufgabe im Ministerium. Dann ist Ernst Pappermann Hauptgeschäftsführer des Deutschen Städtetages geworden und in seinem Büro in Köln kam es an einem Abend im Jahre 1982 zum ersten Treffen des Teams. Wir hatten uns damals sehr rasch über die Grundfragen und das Leitbild des Kommentars abgesprochen, auch über die Aufteilung der Paragraphen. Leider konnte Ernst Pappermann seinen Part nicht leisten und wir haben dann beschlossen, das Buch auch ohne den Vertreter der kommunalen Familie zu dritt zu schreiben. Daß Rolf-Peter Löhr dann schon bei der 3. Auflage als stellvertretender Leiter in das Deutsche Institut für Urbanistik wechselte und wir damit die Trias „Kommune - Wissenschaft - Bundesministerium“ herstellen konnten, war - so gesehen - eine glückliche Fügung.

\section{Das Konzept für die gemeinsame Kommentierung}

\section{Das „Leitbild“}

Wie stellten wir uns das neue Buch vor, was wollten wir erreichen, was war - wie es jetzt heißen würde - das Leitbild? Und wie haben wir die Zusammenarbeit gestaltet? Das war bereits beim ersten Treffen rasch geklärt. Das Städtebaurecht ist wie wenige andere Rechtsgebiete - so der Ausgangspunkt - von der Rechtsprechung geprägt. Es gab schon damals - erste Hälfte der achtziger Jahre - viele Bücher und Kommentare zum Städtebaurecht, wenn auch noch nicht so viele wie heute. Über die Art der Kommentierung - kurz, praxis- und rechtsprechungsorientiert, nicht theorielastig - waren wir uns sehr schnell einig: Verwertung der aktuellen Rechtsprechung, vor allem des Bundesverwaltungsgerichts. Von vertiefenden Darstellungen etwa über die Entstehungsgeschichte der einzelnen Vorschriften und historischen Rechtsentwicklungen, soweit sie nicht für das Verständnis erforderlich sind, sollte der Kommentar freigehalten werden. Eigene abweichende Meinungen soll-

2 Z.B. haben die beiden gemeinsam mit Wolfgang Andriske ein Lehrbuch zum öffentlichen Sachenrecht bei Beck herausgebracht. 
ten - wo aus der Sicht des Autors unabweisbar - angesprochen werden; das Leitbild war aber eine rasche Wissensvermittlung dessen, ,was gilt" Verlag mit dem Großkommentar von „Ernst/Zinkahn/Bielenberg“ und mit Lehrbüchern - wie dem vorzüglichen Buch von Ernst/Hoppe - Werke veröffentlicht, die rechtshistorische und dogmatische Vertiefung leisten sollten.

\section{Die Aufteilung}

Die Aufteilung der Kommentierung konnte, weil wir alle drei bisher nur in Teilbereichen des Städtebaurechts geschrieben hatten - Battis und Löhr sicher bereits am profiliertesten - nicht nach „Vorwissen“ erfolgen. Was war statt dessen der Maßstab? Löhr bot sich z.B. für die Planinhalte ( $§ 5$ bis 9) an, weil er darüber promoviert hatte und im Erschließungsrecht aufgrund seiner juristischen Ausbildung verwaltungspraktische Erfahrungen in einer Großstadt hatte. Das Umlegungsrecht interessierte ihn praktisch und dogmatisch. Battis hatte sich - wenn auch auf der überwölbenden Ebene der „Partizipation“ - mit Aspekten des Bauleitplanverfahrens - habilitiert, also floß ihm gleich das ganze das Bauleitplanverfahren ( $\$ 2$ bis 4) zu. Ich sollte die Grundsätze der Bauleitplanung ( $\$ 1$ ) übernehmen, aber auch die praktisch besonders bedeutsamen Vorschriften der $\S \S 34$ und 35, dies - so die gemeinsame Überlegung - um die Position des Ministeriums in diesen Vorschriften deutlich zu machen.

Die Orientierung an dem, ,was gilt“, dies wiederum fixiert auf die höchstrichterliche Rechtsprechung, ist bei einem Buch, das sich auch an die Wissenschaft wendet, ein riskantes Unternehmen. Der Universitätsprofessor unter uns sollte daher die eher dogmatischen Partien übernehmen (Enteignung, Normerhaltung), vielleicht auch mit dem Hintergedanken, daß er dieses im Wissenschaftlerkreis nicht risikolose Unterfangen abfedert und hier für zusätzliches Profil sorgt. Die Rezensenten haben es wohlwollend akzeptiert.

Der „Rest“ des Gesetzes ist irgendwie nach Menge aufgeteilt worden, nicht ganz gleichgewichtig dem Umfang, aber - so meinen wir - der sachlichen Bedeutung nach. Als wir mit der 2. Auflage die Seitenzahl „2000“, als aus dem Kommentar zum „BBauG“ einer zum „BauGB“ wurde, überschritten, haben wir uns damals rasch darauf verständigt, künftig stets deutlich darunter zu bleiben - und das wurde ungeachtet der diversen Erweiterungen des Rechts namentlich mit dem BauGB-Maßnahmengesetz und dem Einigungsvertrag - so bis zur 10. Auflage ebenso eingehalten wie das „Einfrieren“ der Anteile am bescheidenen Aufwendungsersatz. Damit war auch der Verbleib in der „Gelben Reihe“ umso leichter begründet, die wir der hoch angesehenen, aber eben nicht mehr unbedingt so handlichen „Grauen Reihe“ der Beck’schen Kurzkommentare vorzogen.

\section{Die Abstimmung}

Einen „Herausgeber“ oder formellen „Koordinator“ gab und gibt es nicht. Man arbeitet zusammen und denkt: Zeit hat keiner zuviel, also geht es rasch und unkompliziert zur Sache. Daher Verständigung auf Zitierweisen, Aufbau und Stil, auf 
Abkürzungen und Sachregister und alle unterstützen es ,,auf Ab- und Zuruf“: möglichst keine oder wenige Abstimmungstermine, notfalls ein kurzes Telefonat heute auch die Mail. Hie und da ein kurzer Zuruf. Jeder hatte ,seine“ Teile für sich zu verantworten und sollte auf Binnenkoordination achten. Alle Drei sahen, sehen das Buch auch als ,,ihres“ an. Abstimmungsbedarf gab es daher wenig und er warf keine Probleme auf. Im Vorwort der 1. Auflage - und ähnlich in den Folgeauflagen - ist das redaktionelle Konzept folgendermaßen umschrieben worden:

Trotz der gebotenen Kürze der Kommentierung sind die Abkürzungen auf das allgemein Übliche und Bekannte beschränkt worden. Lediglich bei der Standardliteratur zum Bundesbaugesetz wurde nicht jeweils der volle Titel angeführt; auf das Verzeichnis der hier verwendeten Abkürzungen sei daher besonders hingewiesen. Das Auffinden der gesuchten Erläuterungen erleichtert ein ausführliches Sachverzeichnis; den Erläuterungen der einzelnen Vorschriften ist in der Regel eine Übersicht vorangestellt. Um dem Leser die Systematik der verschiedenen Teile und besonders wichtiger Abschnitte des Bundesbaugesetzes zu erschließen, gehen ihrer Kommentierung - soweit es zweckmäßig erschien - knapp gehaltene Vorbemerkungen voraus, in denen der innere Zusammenhang der Vorschriften dargestellt wird.

\section{Der Name}

Die Reihenfolge der Namen wurde kurz diskutiert - als erster Name Pappermann für die kommunale Familie oder Krautzberger für den ministeriellen Anspruch? Es kam dann das schlagende Argument des Namensträgers, mit „B“ vorne stünden wir bei Literaturverzeichnissen und ähnlichem immer ganz vorn; das sei (auch) verkaufsfördernd. So haben wir uns für die alphabetische Reihenfolge entschieden.

\section{Der Verlag}

Den Kontakt zum Verlag C.H. Beck hatte - das wurde schon erwähnt - von Pappermann vermittelt. Das erste Gespräch mit dem Verlag führte Herr Dr. Hans Dieter Beck gemeinsam mit Herrn Rechtsanwalt Hans-Ulrich Büchting. Die ersten Gesprächsthemen betrafen: Einschätzung des Buchs, nicht weniger der drei neuen Autoren bei Beck, kurze diskrete Erwähnung von Reaktionen der „Umwelt“, die Frage des Umfangs im Vergleich zu anderen Büchern. Den Verleger interessierte auch die Diskussion um die Abschöpfung von Bodenwerten. Hat er - offenbar mit Grausen - an das schöne Grundstück an der Wilhelmstr. 9 gedacht? Und wie steht es mit $\$ 34$ - und dem eigenen Bauvorhaben - bringt der Kommentar dazu genug? Letztlich scheinen Autoren und Texte dem Projekt nicht im Wege gestanden zu sein. In der Zukunft trat der Verleger dann und wann bei Gesprächen mit dem Lektorat hinzu, kurze, präzise Gespräche, andere Buchprojekte kamen ohnehin dazu. Walter Bielenberg hat mir nach 1987 die Mitwirkung in dem im VahlenVerlag erscheinenden Kommentar zum Städtebauförderungsrecht angeboten. Herr 
Rechtsanwalt Burkhard Schulz hat die erneute Erweiterung meiner Beteiligung bei C.H. Beck wesentlich und sehr effizient mitgestaltet als Walter Bielenberg sehr früh einen Nachfolger in der Herausgabe des - so jetzt - Großkommentars „Ernst/ Zinkahn/Bielenberg/Krautzberger" suchte.

\section{E. Das Lektorat}

Zunächst war Herr Rechtsanwalt Büchting unser Gesprächspartner, bis eines Tages Frau Dr. Christiane Dobring am Telefon war - und von da an war sie die wichtigste Persönlichkeit für das Buch und für die Autoren. Herr Rechtsanwalt Burkhard Schulz unterstützte alles in sehr angenehmer Weise als Vertreter des Verlegers. Christiane Dobring, die Lektorin also, wurde die wichtigste Bezugsperson für den Kommentar, die Verläßlichkeit in Person, sie agierte mit Nachdruck, wenn es um Termine, Absprachen und Qualität geht, hörte sich die Klagen über die doch vergleichsweise retardierte Druckerei an und vermittelte Verständnis, aber bewies auch energisches Eingreifen. Fehler, die Autoren ja nie unterlaufen, behandelte sie mit österreichischer Diskretion. Immer ansprechbar, zuletzt - auch der ehrwürdige Verlag C.H. Beck - öffnete sich in den 20 Jahren des Kontaktes behutsam neuen Techniken sogar mit Handy, Anrufbeantworter und Mail und damit sozusagen rund um die Uhr.

Da ich später auch die Herausgabe von „Ernst/Zinkahn/Bielenberg/Krautzberger" übernahm, erweiterte sich der Kontakt. Aber „Autorenkonferenzen“ waren bei „Battis/Krautzberger/Löhr“ gleichwohl die Ausnahme, es bedurfte ihrer nur selten und wenn, dann beschränkte man sich auf ein Mittagessen bei Aigner oder ein Frühstück bei Einstein, waren die drei Autoren doch inzwischen beruflich aus dem großen westdeutschen Bundesland nach Berlin gewechselt. Die Lektorin: diskret, sich zurücknehmend in der mächtigen Männerwelt des Verlags, das Werk mit sichtbarer Freude am Gelingen unterstützend.

\section{F. Die Umwelt}

Der Kommentar traf im Jahre 1984 auf eine schon weitgehend formierte Kommentarlandschaft. Sicher gab es verlagsintern Diskussionen mit wichtigen Autoren. Auch wurde von da und dort versucht, den Umfang des Buchs auf etwa 800 Seiten zu begrenzen; vom Umfang her - das liegt an dem charmanten Format der „Gelben Reihe“ - wirkte das Buch ja schlanker als es ist - zwischen 1800 und 2000 Dünndruckseiten, dabei mit konzentrierter Zitierweise und Verzicht auf die Entstehungsgeschichte u.a. - das gibt genug Raum für eine intensive Abhandlung der Rechtslage wie sonst ein dickleibiger Kommentar. Auch drang manchmal ein Kommentar über uns drei Autoren durch, ob „die“ das wohl zustande brächten oder nur von ,uns“ (also dem Establishment) abschreiben. Aber jung und unverdrossen wie wir waren, gab das eher Anstoß. Insgesamt fühlten wir uns gut aufgenommen. Besonders herzlich empfand ich dies bei den „Großmeistern“ des Städte- 
baurechts Rudolf Stich und Otto Schlichter, die mich bei einer Veranstaltung begrüßten: ,Willkommen in der Familie der Baurechtskommentatoren - nicht Konkurrenten, sondern eine Familie sind wir." Daraus erwuchs dann eine (leider zu kurze) weitere Autorengemeinschaft: Schon kurz nach Erscheinen des „BKL“ schrieben wir zu dritt ein schönes Buch als Kommentar zum damals noch bestehenden Städtebauförderungsgesetz im Heymanns Verlag. Als das Baugesetzbuch 1987 das Bundesbaugesetz und das Städtebauförderungsgesetz zusammenführte, mußte ich mich dann zwischen dem späteren „Berliner Kommentar“ und „BKL“ entscheiden - und Rudolf Stich und Otto Schlichter haben mich verständnisvoll verabschiedet - und vor allem Rudolf Stich und ich haben uns noch später gerne bei Vortragsveranstaltungen getroffen, nicht ohne den Kommentar des anderen gerne und positiv zu würdigen.

\section{G. Die Entwicklung}

\section{Zehn Auflagen}

Zehn Auflagen in weniger als 25 Jahren. Alle drei Jahre eine Neuauflage; bei der fünften, siebenten und zehnten Auflage lagen nur zwei bzw. ein Jahr zwischen Auflagen. Das Städtebaurecht hatte sich allerdings in diesen Jahren sehr intensiv verändert - und das erforderte die relativ häufigen Überarbeitungen. Die intensive höchstrichterliche Rechtsprechung trug das ihre dazu bei. Die späteren Neuauflagen des Buchs waren fast immer durch Novellierungen des Gesetzes evoziert, aber sie wurden auch ausnahmslos zu einer umfassenden Überarbeitung der Kommentierung genutzt, zumindest derjenigen Teile, die - wie im Städtebaurecht sehr ausgeprägt - Gegenstand der Fortentwicklung durch höchstrichterliche Rechtsprechung sind.

\section{Das BauGB 1987}

Die 2. Auflage (1987) war durch die schon erwähnte umfassende Neukodifikation des Städtebaurechts im neuen „Baugesetzbuch“ veranlaßt. Das Städtebaurecht wurde erheblich umgestaltet. Die Regelungen des Sanierungs- und Entwicklungsmaßnahmerechts aus dem Städtebauförderungsgesetz wurden mit dem Bundesbaugesetz zusammengeführt. Darüber hinaus setzte das neue BauGB eine Reihe neuer Akzente zugunsten einer Konsolidierung der Siedlungsräume

Die Regierungserklärung der 2. Regierung Helmut Kohl im Frühjahr 1983 gab den Anstoß für die neue Gesetzgebung, wobei das „Bundesbaubuch“, wie es der damalige Regierungschef gerne nannte, vor allem der Entbürokratisierung einerseits und - so berichtete der damalige Bundesbauminister Oscar Schneider - andererseits dazu beitragen solle, in Deutschland eine baukulturelle Qualität zu sichern wie sie der Kanzler bei seinem Besuch in Assisi habe feststellen können. Oscar Schneider seinerseits war es ein Anliegen, den Grundsatz der „Baufreiheit“ - möglichst als Präambel - dem Baugesetzbuch, das er sich im übrigen als ,Jahrhundert- 
gesetz" vorstellte, voranzustellen. Die Länder drängten zugleich auf eine Verlagerung von Zuständigkeiten auf sie: Abbau der Mischfinanzierungen und Freigabe bundesrechtlicher Kompetenzen an die Länder.

Die Ergebnisse zu alledem fielen ernüchternd aus. Was den Abbau von Bundeskompetenzen betraf, „kippte“ im federführenden Bauausschuß des Deutschen Bundestags die Stimmung der damaligen Koalition, als der Sprecher der Bauministerkonferenz der Länder die Freigabe des $§ 126$ Abs. 3 BBauG/BauGB verlangte, weil die Bestimmung, jeder Eigentümer habe sein Haus mit einer Hausnummer zu versehen, als unerträgliche Gängelung des Bundes anzusehen sei. Hausnummern brauche es doch und was denn damit gewonnen sei, wenn dies statt in einem Bundesgesetz in (damals) elf Ländergesetzen geregelt würde, so entrüstete sich ein Sprecher der Koalition. So kippte auch die von allen Verbänden und Experten abgelehnte Freigabe diverser anderer Regelungsbereiche (u.a. Enteignung, Erschließung) und die Mischfinanzierung wurde über eine Überleitungsbestimmung nachhaltig abgesichert.

Die Forderung, durch Verschlankung des Baurechts zur Entbürokratisierung beizutragen, hielt sich als Grundton auch bei allen späteren Novellen bis hin zur BauGB-Novelle 2007. Das ist nur zu verständlich, verbinden sich mit dem Bauen doch in besonderem Maße vielfältige Regelungen, Planungs- und Genehmigungserfordernisse und Behördenzuständigkeiten. Es wird sicher auch bei künftigen Gesetzgebungen immer wieder angezeigt sein, den Garten der BauGB-Paragraphen, dem es an Nachwuchs nicht mangeln dürfte - gestern wuchsen die Windräder nach, heute die Biomasseanlagen und erwarten ihre Regelung im BauGB -, kritisch auf Wildwuchs zu durchforsten. Aber der „Befreiungsschlag“ wird hier nie gelingen, weil im Bauplanungsrecht am allerwenigsten ,das“ Problem liegt. Auch der frühere Bundeskanzler Helmut Schmidt macht in „DIE ZEIT“ periodisch das „Baubuch“ mit seinen fast 2000 Paragraphen für die Situation der deutschen Wirtschaft verantwortlich, dabei hat nicht einmal der Kommentar „BKL“ 2000 Seiten aufzuweisen. Aber gemeint ist ja etwas anderes und das war bei Oscar Schneider so zutreffend wie bei Helmut Schmidt: Das baurechtliche Umfeld ist in der Zeit seit Schaffung des Bundesbaugesetzes so in die Höhe geschossen, daß es das Baurecht selbst schon längst zu überwachsen scheint. § 34 BauGB über das Baugeschehen im nicht-beplanten Innenbereich z. B. steuert etwa 50\% des Baugeschehens und umfaßt in seinem Hauptanwendungsfall nach wie vor nicht mehr als einen Satz: $§ 34$ Abs. 1 S. 1. Aber 1960 gab es weder ein Bundesnaturschutzgesetz und 16 Ländergesetze hierzu, keine Umweltgesetzgebung, also auch kein BImSchG, keine Denkmalschutzgesetze sowie keine europäischen Regelungen von FFH bis UVP und UP.

\section{Der Einigungsvertrag 1990}

Die 3. Auflage (1990) wurde durch zwei unterschiedlich gewichtige, aber in innerem Zusammenhang stehende Gesetzgebungen veranlaßt: Das am 1. Juni 1990 in Kraft getretene „Wohnungsbauerleichterungsgesetz“, ersetzte oder ergänzte mit dem „Maßnahmengesetz zum BauGB“ für einen Zeitraum von fünf Jahren eine 
Reihe von Vorschriften des Baugesetzbuchs. Das BauGB selbst - eben noch als Jahrhundertwerk bestaunt - sollte nicht schon nach drei Jahren geändert werden. Das „BauGB-Maßnahmengesetz“ verfolgt vor allem das Ziel, zeitlich befristete Erleichterungen des Planungs- und Baurechts zu schaffen, damit Wohnbauland zügig und ausreichend ausgewiesen und die Zulassung von Wohnbauvorhaben erleichtert wird. Anlaß dazu gaben nicht zuletzt die erheblichen Zuwanderungen aus Mittel- und Osteuropa (,Um- und Aussiedler“ war der Terminus) seit Ende der achtziger Jahre - Vorboten der Zeitenwende. Und diese stand Pate für die zweite Änderung des Jahres 1990: Mit dem Beitritt der DDR zur Bundesrepublik Deutschland galt das BauGB auch in den Ländern Brandenburg, MecklenburgVorpommern, Sachsen, Sachsen-Anhalt, und Thüringen sowie in Berlin (Ost), allerdings mit einer Reihe von übergangsweise geltenden Modifizierungen, die sich aus dem Neuaufbau einer kommunal verantworteten, rechtsstaatlichen städtebaulichen Planung erklären. Diese Regelungen waren im damaligen § 246a BauGB zusammengefaßt. Später zeigte sich, daß eine Reihe wesentlicher Elemente dieser Sonderreglungen auch in die nord-, west- und süddeutschen Länder übernommen werden konnten. Insgesamt zeigte sich aber rasch, daß die Strukturen des im BauGB kodifizierten Bauplanungsrechts der Struktur deutscher Städte, den Traditionen des Planens, Erschließens und Bauens, aber auch der Ausbildung von Planern und Architekten in einem Maße entsprach, die einer letztlich recht unkomplizierten Übernahme des BauGB den Weg bereitete und schon 1993 in einem ersten Schritt, endgültig dann 1998 die Sonderregelungen für die östlichen Länder sogar insgesamt entbehrlich machte. Dies hing sicher ganz maßgeblich auch mit dem nicht überall einfachen, aber alles in allem doch kraftvollen und auf breiten Konsens der Bevölkerung gestützten Wiederaufbau der in Deutschland so traditionsreichen kommunalen Selbstverwaltung zusammen, zu deren Kernaufgaben stets auch die Städtebaupolitik gehörte.

\section{Neue städtebaurechtliche Strukturfragen - die Novellen 1983 und 1998}

Mit dem am 1. Mai 1993 in Kraft getretenen „Investitionserleichterungs- und Wohnbaulandgesetz" wurde eine Reihe neuer Akzente gesetzt, die - wie sich heute zeigt - weit in die Zukunft der städtebaurechtliche Entwicklung wirkten. Die vierte Auflage (1993) setzte diese Änderungen um. Zunächst konnten in einem ersten Schritte auf einige der Sonderregelungen für die östlichen Bundesländer verzichtet werden. Im Gegenzug wurden aber vor allem zwei dieser bisherigen „Sonderregelungen“ in allen 16 Ländern eingeführt: der städtebauliche Vertrag und der Vorhaben- und Erschließungsvertrag. Mit der Kodifizierung des städtebaulichen Vertragsrechts begann geradezu ein „Siegeszug“ dieser - was den städtebaulichen Vertrag betrifft ja gesetzgeberisch nichts als das geltende Recht in Gesetzessprache fassenden - konsensualen Verfahren. Zunächst noch im BauGBMaßnahmengesetz befristet verankert übernahm das am 1. Januar 1998 in Kraft getretene „Bau- und Raumordnungsgesetz 1998“ diese Bestimmungen in das BauGB selbst, und zwar - mit $\$ \S 11$ und 12 - an recht hervorgehobener Stelle. Im Grunde war dieses Regelwerk eine der beiden wichtigsten Reformen im 
Städtebaurecht zu einer weitreichenden und wie sich zeigte nachhaltigen strukturellen Vereinfachung des Bau- und Planungsrechts; politisch waren beide Reformen von der damaligen Bundesbauministerin Irmgard Schwaetzer gestaltet und vermittelt worden: Vertrag anstelle Satzung oder sonstiger ,einseitiger“ kommunaler Gestaltung. Rechtsfrieden, Akzeptanz und Verbreiterung der städtebaulichen Gestaltungsmöglichkeiten wurden damit gemehrt. Daß heute in immer mehr Städten und Gemeinden städtebauliche Verträge aus der Städtebaupolitik nicht mehr wegzudenken sind, ist auf diese gesetzgeberischen Entscheidungen des Jahres 1993 zurückzuführen.

Die andere, gleichfalls weit in die Gegenwart hineinwirkende strukturelle Vereinfachung war der Einstieg in die Neuregelung des Verhältnisses von Umweltund Naturschutzrecht zum BauGB. Das moderne Umwelt- und Naturschutzrecht begann sich erst in den siebziger Jahren $\mathrm{zu}$ formen. Anfang der neunziger Jahre war unklar, ob sich angesichts der starken räumlichen Bezüge dieses ,jungen“ Rechtsbereichs ein „zweiter Pfad“ räumlicher Planung erschließt - so die Tendenz in einigen Landesnaturschutzgesetzen und bei manchen Vordenkern im Umweltbereich - oder ob ein Weg gefunden wird, Umwelt- und Naturschutzrecht nicht anders als die übrigen Fachbereiche (Gewerbe, Wohnen, Gemeinbedarfsanlagen usw.) - hinsichtlich seiner Raumansprüche in das städtebauliche Planungsrecht zu verankern. Letzteres löste bei der Umweltpolitik erhebliche Empfindlichkeiten aus, die politisch erst in einem umfangreichen Vermittlungsverfahren zugunsten der Integration der naturschutzrechtlichen Eingriffsregelung nach dem BNatSchG in die Entscheidungsprinzipien und Verfahren der Bauleitplanung darum wurde damals die Kontroverse geführt - entschieden wurde.

Der damalige Bundesumweltminister Klaus Töpfer erreichte damals, daß dieser „Baurechtskompromiß“ gesetzlich im BNatSchG und nicht im BauGB verankert wird; im „Bau- und Raumordnungsgesetz 1998“ - zugleich wesentlicher Anlaß für die sechste Auflage (1997); die fünfte Auflage (1995) war eine Zwischenauflage, die wegen der erfreulichen Aufnahme des Kommentars, aber auch der Konsolidierung der Rechtsprechung zu den seit 1990 ergangenen Rechtsänderungen erforderlich wurde - hat der Bundesbauminister Klaus Töpfer politisch abgesichert, $\mathrm{da}$ die Regelungen in das BauGB übernommen wurden. Zugleich wurden die Weichungen dafür gestellt, daß auch weitere Umweltbelange hinsichtlich ihrer städtebaulichen Dimension nach den Verfahren und in Integration in deren Entscheidungsmechanismen eingebunden werden können.

Das „BauROG 1998“ stellte den Kommentatoren die Aufgabe, eine Reihe weiterer struktureller Änderungen aufzugreifen: Von der erwähnten Verankerung der konsensualen Verfahren in $\S \S 11$ und 12 BauGB, über ein neues Konzept der baurechtlichen Beurteilung von Umwidmungen ehemals landwirtschaftlich genutzter Gebäude (\$35 Abs. 4 S. 1 Nr. 1) bis hin zur Neuakzentuierung des Verhältnisse zwischen Gemeinde und Staatsaufsicht. Nach dem Bundesrecht wurden städtebauliche Satzungen weitgehend von der präventiven Kontrolle freigestellt. Diese und weitere Änderungen waren z. T. nur um den Preis von dann vielfach kritisierten „Länderabweichungsregeln“ zu erzielen, die freilich in der Praxis im wesentlichen keine oder nur marginale und befristete Bedeutung erlangten, besteht im 
Städtebaurecht doch offenbar kein praktisches Bedürfnis nach regionaler Ausfächerung des ohnehin auf individuelle kommunale Ausformung angelegten Rechtsgebiets.

Die Frage: „Änderung des BauGB oder nicht“, die noch 1990 zum BauGBMaßnahmengesetz führte, stellt inzwischen niemand mehr. Im Vorwort der vierten Auflage hieß es dazu: „Eine Würdigung dieser gesetzlichen Neuregelungen zeigt, wie unrealistisch die - freilich durchaus verständliche - Vorstellung ist, das Städtebaurecht ließe sich für längere Zeit aus dem Gesetzgebungsprozeß heraushalten. Gerade weil städtebauliche Planungen und Maßnahmen unmittelbar mit wirtschaftlichen, gesellschaftlichen und ökologischen Sachverhalten verknüpft sind, wirkt die Dynamik dieser Bereiche auf die künftig im europäischen Binnenmarkt noch stärker EG-rechtlich dirigierte nationale Gesetzgebung zurück.“

\section{Umwelt- und Europarecht - die Novellen 2001, 2004 und 2007}

Die siebente bis zehnte Auflage waren in besonderer Weise von den Entwicklungen im Umwelt- und Europarecht geprägt. Die siebente Auflage (1999) war wegen der notwendigen Anpassung an Änderungen im Bundesnaturschutz und das neue Bundes-Bodenschutzgesetz, aber auch wegen des recht erfreulichen Erfolges der Vorauflage erforderlich. So hieß es im Vorwort: „Von der Europäischen Union werden ebenso neue Initiativen erwartet wie von der nationalen Gesetzgebung, nicht zuletzt von den Arbeiten an einem Umweltgesetzbuch." Letzteres war eine zu optimistische Erwartung, ersteres prägte in den Folgejahren die städtebaurechtliche Entwicklung wie kein anderer Einfluß. Die achte Auflage (2001) ist erforderlich geworden, weil das am 3. August 2001 in Kraft getretene Gesetz zur Umsetzung von EG-Richtlinien zum Umweltrecht zu einer weitreichenden Änderung der Anforderungen an die Umweltverträglichkeitsprüfung (UVP) innerhalb des Bebauungsplanverfahrens führte. Neben inhaltlichen und verfahrensmäßigen Anpassungen an eine UVP hat die Neuregelung die Vorhabenarten, für die eine UVP europarechtlich vorgesehen ist, erheblich ausgeweitet.

Diese UVPG-Novelle 2001 wurde - wegen der verspäteten Umsetzung der entsprechenden EG-Regelungen durch das UVPG - erst zu einem Zeitpunkt wirksam, zu dem sich ein neues Konzept des EU-Umweltrechts für städtebauliche Planung abzeichnete. So war sie letztlich nur ein Vorläufer der Gesetzesänderungen von 2004. Die neunte Auflage (2004) setzte - so jedenfalls der unabweisbare Anlaß - mit dem am 20. Juli 2004 in Kraft getretenen „Gesetz zur Anpassung des Baugesetzbuchs an EU-Richtlinien (Europarechtsanpassungsgesetz Bau - EAG Bau)“ vor allem die ,Richtlinie zur Umweltprüfung von Plänen und Programmen“ um. Die durch die Novelle 2001 eingeführte Umweltverträglichkeitsprüfung für bestimmte Bebauungspläne war in der nunmehr geltenden umfassenden Regelung über die Umweltprüfung aller Bauleitpläne aufgegangen. Bauleitpläne Flächennutzungspläne ebenso wie Bebauungspläne - sind einer Umweltprüfung $\mathrm{zu}$ unterziehen, was besondere Beteiligungen von Öffentlichkeit und Behörden erfordert, den Umweltbericht für jeden Bauleitplan als Teil der Begründung, eine Darstellung, wie die Beteiligungen und der Umweltbericht im Plan berücksichtigt 
wurden und nicht zuletzt ein neues Überwachungsverfahren (,Monitoring“). In einer Anlage zum BauGB sind (an das EU-Recht angelehnte) Konkretisierungen für den Umweltbericht enthalten.

Im Vorwort zur neunten Auflage wird diese Entwicklung angesprochen: „, Auch das Städtebaurecht ist wegen der Ausfüllung der Umweltkompetenz der Europäischen Union inzwischen also voll in den main-stream auch des europäischen Verwaltungsrechts und seiner Akzentuierung des Verwaltungsverfahrens eingelenkt. Diese Entwicklung scheint noch keineswegs abgeschlossen, arbeitet die Kommission doch an einer weiteren Ausformung des Umweltrechts auch in seiner räumlichen Ausstrahlung. In den Verfahrensvorschriften des BauGB ist der Gesetzgeber - schon zur Vermeidung eines Nebeneinander EU-rechtlich-bestimmter und national-bestimmter Verfahrensregeln - dem europäischen Wegweiser gefolgt. Ein sich ausformendes, stark verfahrensbestimmtes, Transparenz sicherndes europäisches Verfahrensrecht prägt zunehmend das Verfahrensrecht der Bauleitplanung. Auch inhaltlich Anforderungen könnten hinzukommen. Hier können politische Gestaltungsmöglichkeiten einer Modernisierung der Rechtsordnung, einer Vereinfachung im Hinblick auf die sich abzeichnende europäische Rechtsentwicklung, der Vermeidung ,,zweier Rechtskulturen“ liegen, die freilich mehr verlangen als Sonntagsreden gegen die am Freitag danach im Bundesrat beschlossenen europäischen Gängelungen, das Subsidiaritätsprinzip als Waffe gegen Modernisierung führend, statt offensiver Gestaltung der möglichen und eben notwendigen Innovationen."

An dieser Einschätzung hat sich auch nichts durch die BauGB-Novelle 2007 umgesetzt in der zehnten Auflage (2007) - geändert, die freilich mit dem Ziele einer Erleichterung der Innenstadtentwicklung die gerade (2004) eingeführten europarechtlichen Vorgaben auf Unabweisbares zurückführte.

\section{H. Einige Erfahrungen}

\section{Wem macht Kommentarschreiben eigentlich Freude?}

Mich selbst kostetet der Gedanke, einen Kommentar zu schreiben, anfangs erhebliche Überwindung, und zwar aufgrund der Freundschaft mit dem Sohn eines maßgeblichen Beck-Autors: Vom hoch geachteten langjährigen Palandt-Herausgeber, dort auch Bearbeiter des Erbrechts und Verfasser weiterer Bücher, Theodor Keidel, war mir nur zu gut in Erinnerung, daß er sich nach dem Abendessen erhob, um in seinem Arbeitszimmer zu kommentieren. „So etwas nie“ dachte ich mir damals. Und als mir - war es vor der Entscheidung zu „Battis/Krautzberger/ Löhr" oder während der Entstehungszeit - Werner Bielenberg eine Mitwirkung im „Ernst/Zinkahn/Bielenberg“ anbot, habe ich wohl auch wegen dieser gerade$\mathrm{zu}$ „traumatischen“ Erinnerungen an München fast schon entsetzt abgelehnt. Später hat sich das dann ja alles etwas verändert. Mein Beitrag entstand dann zunächst in einem Urlaub in Holland am Meer, bevor die Familie aufstand und später - das dauerte mehr als ein Jahr - regelmäßig ab 22 Uhr bis morgens 1 Uhr und später. Darauf noch ein Sommerurlaub mit Frühschicht (die Kinder waren 
immer noch kleine Langschläfer, meine liebe Frau ohnehin) und das Buch war geschrieben.

Dann das erste Manuskript: Es ist endlich fertig, ein dickes Paket - morgen früh zur Post damit - abends sind wir eingeladen - daß dem Manuskript nur ja nichts passiert - was hat man da schon für Geschichten gehört über fertige Manuskripte, Einbrüche, Brände! (Bitte ruhig darüber lachen: Ich habe es in den Keller zwischen die Weinflaschen gelegt - neurotische Ängste, na gut.) Lob der technischen Entwicklung: Die 9. Auflage wurde am PC hergestellt - erstmals nicht diktiert und abgetippt, sondern selbst am PC geschrieben - und daß der Text per Mausklick zu Dr. Christiane Dobring flog, das war eine Erleichterung, namentlich nach der Sicherung auf einer CD-Rom und auf dem Laptop dazu.

\section{Kommentarschreiber im Bundesministerium}

Kommentatoren, die - wie der Verfasser - auch persönlich für das kommentierte Gesetz verantwortlich sind, müssen mit der Meinung leben, sie würden die Gesetzesnovellen zeitlich mit dem Stand des Verkaufs der Vorauflage steuern. (,,Was wären wir ohne den Gesetzgeber" rief der im Bauplanungsrecht profilierte Autor Bernhard Stüer anläßlich des EAG Bau 2004.) Ob Bundestagsabgeordnete oder Bundesminister - immer kam dann einmal zum Abschluß der parlamentarischen Beratungen die Bemerkung, der Kommentar sei schon fertig - oder könne man noch einen Antrag stellen? Den damalige Bundesbauminister Klaus Töpfer konnte ich z. B. vor dem Vermittlungsverfahren zum Bau- und Raumordnungsgesetzes 1998 (die sechste Auflage stand an) nicht von der Überzeugung abbringen, ich hätte das Manuskript schon fertig - und zwar unter Berücksichtigung der von meinen Mitarbeitern und mir $\mathrm{zu}$ formulierenden Kompromißanträge für den Vermittlungsausschuß. Alle wußten, das war ein Spiel - irgendwie ist den Politikern die Ministerialbürokratie zwar lieb, aber auch etwas unheimlich. Aber wenn sie von dritter Seite wahrnehmen, daß es der Reputation dient, dann kann das ja auch das politische Anliegen fördern und so arrangiert sich alles, umso mehr wenn man - wie bei „B/K/L“ - mit Ulrich Battis den angesehenen Hochschulprofessor und mit Rolf-Peter Löhr einen ,Vertrauten“ der kommunalen Familie im Autorenteam wahrnimmt.

Natürlich ist das Kommentare-Schreiben auch ein Ausgleich für den „Schock“, den jeder „Gesetzesschreiber“ eines Tages erleidet, sobald nämlich die von ihm entworfene und dann liebevoll betreute Novelle eines Tages im Bundesgesetzblatt steht. Bis dahin hat man ja vielfältige Möglichkeiten, den Gesetzestext von der Entstehung im Ministerium bis zum Ende der parlamentarischen Beratungen aktiv $\mathrm{zu}$ begleiten und $\mathrm{zu}$ gestalten, selbst oder aber über hilfreiche Hände bei den Ländern, den Abgeordneten, mitunter auch der jeweils regierungsoppositionellen, Verbänden und Experten - zuletzt auch noch bis in das stilistische Detail hinein. Aber dann steht alles irgendwann im ,BGBl.“. Und man kann daran plötzlich nichts mehr ändern. Das Kind ist erwachsen. Das ist die Stunde des Kommentators: Es kann weiter interpretierend geformt, ja auch veredelt werden. Und: Mögen die eigenen Gesetze auch fehl- und tadellos sein (jedenfalls muß man 
das ja so vermitteln), so kennt man doch die Passagen, die besonderer interpretatorischer Zuwendung bedürfen oder die das Ergebnis von Kompromissen waren, zu denen man rasch die Meinung der Gesetzesschreiber - respektive der Bundesregierung - zur möglichst ,herrschenden“ Meinung machen sollte. Kommentare schreiben - so gesehen die Überbrückung der Entzugsfolgen bis zur nächsten Novelle?

Das wäre Wasser auf die Mühlen des erwähnten politischen Umfelds - nur Ulrich Battis und Rolf-Peter Löhr stünden ohnehin frei gezeichnet. Freilich: Auch sie sind weit über das fachlich-wissenschaftliche Interesse an der Rechtsentwicklung interessiert und wirken an ihr profiliert und - so jedenfalls das Urteil der Fachwelt und der Gesetzgebungstheorie - Maßstab setzend mit. Ulrich Battis betreute wissenschaftlich und als Mitglied zweier Expertenkommissionen des Bundesministeriums zur Gesetzesvorbereitung die - nach ihren Vorsitzenden schlagwortartig genannte - „Schlichter-Kommission“ zur Vorbereitung des BauROG 1998 und die „Gaentzsch-Kommission“ zur Vorbereitung des EAG Bau 2004. Die hohe Akzeptanz dieser beiden Novellen im politischen Raum wäre sicher so nicht zu erreichen gewesen, wenn die fachliche Meinungsbildung dadurch nicht so weit vorgeklärt war, daß politisch unterschiedliche Bewertungen nicht ausgeschlossen, aber der außer politischer Bewertung stehende Stoff doch sehr präzise bezeichnet war. Die politische Diskussion war somit sachlich vorstrukturiert.

Rolf-Peter Löhr begleitete als stellvertretender Leiter des Deutschen Instituts für Urbanistik (DIFU) u. a. die beiden vom DIFU durchgeführten Planspiele zu diesen beiden Gesetzen, aber auch zur UVPG-Novelle 2001. Planspiele wurden auch schon zum Baugesetzbuch 1987 und zu früheren Novellen durchgeführt. Damit ist ein Gesetzgebungsstandard erreicht worden, der das Städtebaurecht von anderen Gesetzgebungsbereichen unterscheidet in Theorie und Praxis nicht selten als vorbildhaft zitiert wird und vor allem auch den Effekt hatte, das Gesetz sozusagen vorab einer Fehlerprüfung unter Praxisbedingungen zu unterziehen, die wichtigen Meinungsträger im vorpolitischen Raum, namentlich die Kommunalen Spitzenverbände auf einer gemeinsame Linie zu verständigen und nicht zuletzt bei den Parlamentariern ganz wesentlich das Verständnis der Regelwerke zu unterstützen und die politischen Entscheidungsbedarfe zu klären.

Aber zurück zum Anfang: Am Anfang stand der Drang, sich in der Materie besser auszukennen. Neugier, Schreibfreude, natürlich auch etwas Freude, seinen Namen zu lesen, erst recht in einer Gerichtsentscheidung und damit zur Weiterentwicklung des Rechts auch kommentierend beitragen zu können. So gesehen ein Interesse gleichermaßen an fachlicher, wissenschaftlicher und gestaltender Arbeit. Allein: ,Wie man es erzählt, ist es nicht gewesen“, an diesen Satz von Christa Wolf erinnert Rolf-Peter Löhr. 